\title{
An Unequal Error Protection (UEP) for JPEG2000 Image using Low Density Parity Check (LDPC) and Hamming Codes
}

\author{
M. Hammad*, G. M. Hamad*, Fathy M. Ahmed*, and K. H. Moustafa ${ }^{*}$
}

\begin{abstract}
Joint Photographic Experts Group (JPEG 2000) is an image compression standard which is recently became one of the most used techniques in image transfer, especially for satellite images. Unequal Error Protection (UEP) coding techniques can be used to increase image transfer immunity against channel noise. In UEP, the important parts of the image are protected using stronger codes than that used for the other parts. This paper proposes an UEP scheme by applying Low Density Parity Check Code (LDPC) for protecting the Region Of Interest (ROI) and headers (markers and marker segments) and Hamming code for protecting compressed data bits. A digital communication system which incorporates a Binary Phase Shift Keying (BPSK) as a common modulation technique is built for the purpose of verifying the proposed UEP scheme performance. The performance of the proposed UEP scheme is compared subjectively and objectively, to validate its superiority, with the traditional Equal Error Protection (EEP) scheme.
\end{abstract}

\section{Keywords}

Unequal Error Protection, UEP, low density parity check code, LDPC, joint photographic experts group, JPEG2000, and Image transmission.

\section{Introduction}

Satellite images are usually composed of a large number of pixels. Transmitting such huge images over bandlimited channels in real-time is a challenge. Alternatively, the original image may be transformed using a global transformation technique into a set of transform coefficients, which are then quantized and coded. Those coefficients could be coarsely quantized in order to reduce the transformed image size with little image distortion. However, the resulting encoded image becomes more sensitive to channel noise which necessitates of using error control techniques which increase the reliability of the channel by reducing the information rate.This can be accomplished by adding redundancy to the information being transmitted. This process leads to a longer coded symbols vector than the actual information. These processes are needed for designing practical image communication systems.

A communication system is used to send and receive the information from a source to a user. The basic block diagram of a digital communication system is shown in Fig.1.The digital source represents the information to be transmitted over the channel to the end user. The source coding (image compression) is used in limited bandwidth requirement applications. Different image compression algorithms may be used. In the present work, the JPEG 2000 which is one of the most used techniques in image transfer is considered. The channel coding provides a reliable communication system by introducing redundancy bits to the actual information. A proposed UEP coding scheme is used in building the communication system. Modulation is performed to transmit the channel encoded digital information over a band pass channel by changing a fixed frequency limited carrier signal in accordance with the channel encoded digital information. A communication channel is used to transmit modulated information from a transmitter to a receiver. In a receiver section the inverse process takes place. 


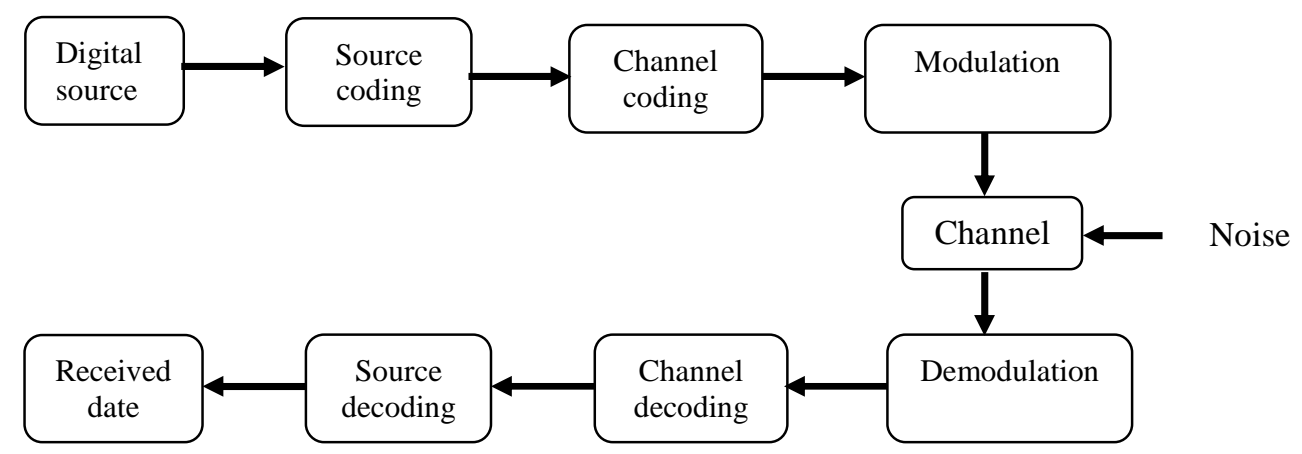

Fig. 1 Basic block diagram of digital communication system

The rest of this paper is organized as follows: after the introduction, section 2 describes the JPEG2000 image coder. Section 3 descries the proposed UEP scheme. Section 4 introduces the performance measure which shall be used for evaluating the proposed scheme. Simulation resultsare presented in section 5. Finally, conclusion comes in section 6.

\section{JPEG2000 Image Coder}

The transformation technique used in JPEG2000 is the Discrete Wavelet Transform (DWT). The first operation is to (optionally) partition a source image into a number of rectangular non overlapping blocks called tiles. Then DWT is applied to each tile which essentially analyzes an image by decomposing it into subbands at different levels of resolution. The first level of decomposition consists of four subbands LL1, LH1, HL1, and HH1. The LL1 subband is the lowest resolution of the tile and is a down sampled low resolution representation of the original tile component. The LL1 subband can be further decomposed by applying DWT. This process can be repeated to obtain different resolution levels. Then, each resolution of each tile component is further partitioned into precincts. Within every subband, each precinct contributes one packet to the code-stream of the image. Entropy encoding is used to further subdivide the precincts into code-blocks. Each code-block is then decomposed into a number of bit-planes. Finally the coder scans through the bit planes within three coding passes. Each of the coding passes collects the relevant information about the bit-plane data. The encoder uses this information to generate a compressed bitstream which consists of two fundamental types of data: (1) compressed data in the form of packets and (2) syntactical data in the form of markers and marker segments that define the characteristics of the image and delimit the code-stream. Certain markers and marker segments are combined to form headers.Each header (Markers/Markers segment) and compressed data is represented with hexadecimal value. For example, Start Of Codestream(SOC) is represented with (0xFF4F), image and tile size (SIZ) is represented with (0xFF51), Coding style Default (COD) is represented with (0xFF52), Quantization Default (QCD) is represented with (0xFF5C), Region Of Interest (RGN) is represented with (0xFF5E), the Start Of Title (SOT) is represented with (0xFF90) and Start Of Data (SOD) is represented with(0xFF93) and etc... [1-7].

JPEG2000 has the capability to define regions of interest of any shape and size and code the selected regions with better quality than the rest of the image and is coded earlier than the rest of the image. ROI coding can be accomplished by encoding the quantized wavelet coefficients corresponding to the ROI with a higher precision relative to the background, e.g. Max-shift method can be used to code the ROI by scaling up the ROI coefficients or scaling down the background coefficients [3], [6], and [8]. 


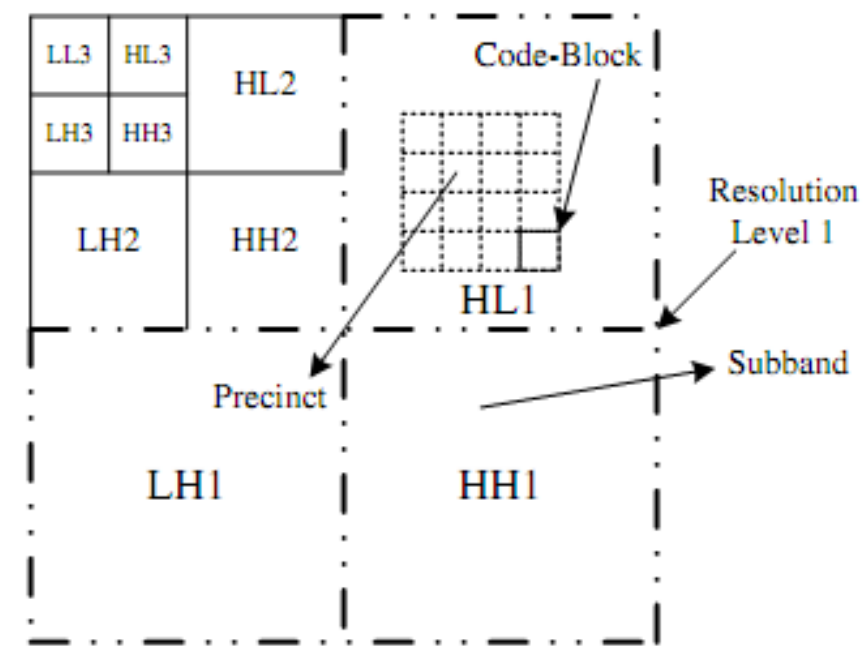

Fig. 2. Components of a JPEG2000 transformed image.

Fig.2 illustrates a 3 layer decomposition of a source image using DWT and its partitioning into four resolution levels, subbands, precincts, and codeblocks.

\section{A Proposed UEP scheme}

UEP is one of the appropriate techniques used for protecting scalable bitstreams against bit corruptions. The idea behind UEP is driven by the fact that different portions of scalable bitstreams have different impact on the quality of the decoded image. The UEP scheme exploits the hierarchical structure of the scalable coded bitstreams and assigns higher protection to the more important parts [9-10].

In this paper, a proposed UEP scheme is incorporated in a digital communication system as shown in Fig. 3. The proposed UEP scheme takes the hierarchy of information for JPEG2000 image into consideration and apply LDPC with rate (1/2) for protecting the ROI and headers (markers and marker segments) while it uses Hamming $(7,4)$ code for protecting compressed data bits over a White Gaussian Noise (WGN) channel. The digital communication system also includes appropriate BPSK modulation.

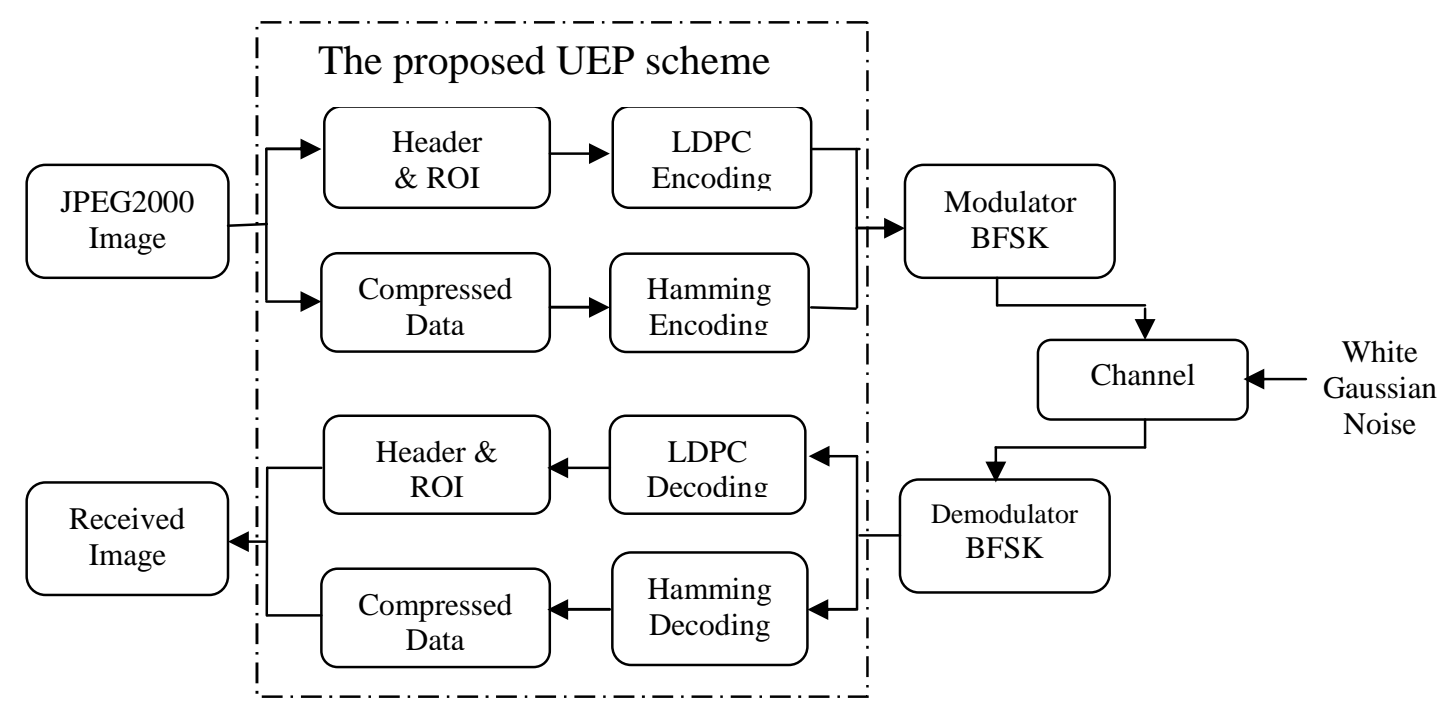

Fig. 3. Incorporating the proposed UEP in digital communication system. 


\subsection{Low Density Parity CheckCode}

LDPC codes are linear block codes defined by a very sparse parity check matrix $\mathbf{H}_{\mathrm{LDPC}}$. A code with rate $\mathrm{R}=\mathrm{K} / \mathrm{N}$ is defined by the number of input bits $\mathrm{K}$ in A block and the number of output bits $\mathrm{N}$. Matrix $\mathbf{H}_{\mathrm{LDPC}}$ is required to be full rank with dimensions $\mathrm{M} \times \mathrm{N}$, where

$\mathrm{M}=\mathrm{N}-\mathrm{K}$. Regular LDPC codes are defined by a constant row weight of $\mathrm{W}_{\mathrm{r}}$ and a column weight $\mathrm{W}_{\mathrm{c}}$ where $\mathrm{W}_{\mathrm{r}}=\mathrm{W}_{\mathrm{c}} \times \mathrm{N} / \mathrm{M}$. Therefore, $\mathbf{H}_{\mathrm{LDPC}}$ has a small number (density) of ones, giving the code name. If the number of one's per column or row is not constant, then the code is an irregular code. In this paper, we consider regular LDPC codes. binary $(\mathrm{N}, \mathrm{k})$ LDPC code is described by a sparse parity check matrix of size $(\mathrm{M} \times \mathrm{N})$, where $\mathrm{M}$ is the number of paritychecks corresponding to the parity-check nodes in a bipartite graph, and $\mathrm{N}$ is the number of variable nodes corresponding to the encoded symbols[11-13].

The encoding of LDPC codes is the same as that of common block codes. But it has a special decoding method, an iterative probability algorithm known as Sum-Product Algorithm (SPA) or belief propagation. At each iteration of decoding, each bit node gets probability message from all the check nodes connected to it, and sends messages back to these check node, which will receive messages from the connected bit nodes and transfer the processed messages back to these bit nodes.

Before discussing the SPA algorithm, we introduce some terms that will be used throughout the discussion of the SPA algorithm:

For the $j^{\text {th }}$ row in a $\mathbf{H}$ matrix, the set of column location of the 1's is given by $R_{j}=\left\{i: h_{j i}=1\right\}$. The set of column locations of the 1's excluding location $i$ is given by $R_{j / i}=\left\{i^{\prime}: h_{j i}=1\right\} \backslash\{i\}$.

For the $i^{\text {th }}$ column in a $\mathbf{H}$ matrix, the set of row locations of the 1 's is given by $c_{i}=\left\{j: h_{j i}=1\right\}$. The set of row locations of the 1's excluding the location $j$ is given by $c_{i \backslash j}=\left\{j^{\prime}: h_{j^{\prime} i}=1\right\} \backslash\{j\}$.

$q_{i j}(b)$ : Message (extrinsic information) to be passed from variable node $v_{i}$ to check node $f_{j}$ regarding the probability that $c_{i}=b, b \in\{0,1\}$, as shown in Figure 3.4. It equals the probability that $c_{i}=b$ given extrinsic information from all check nodes, except node $f_{j}$.

$r_{j i}(b)$ : Massage to be passed from check node $f_{i}$ to variable node $v_{i}$, which is the probability that the $j^{\text {th }}$ check equation is satisfied given bit $c_{i}=b$ and the other bits have separable (independent) distribution given by $\left\{q_{i j}\right\}_{j^{\prime} \neq j}$

$$
\begin{aligned}
& Q_{i}(b)=\text { the probability that } c_{i}=b, b \in\{0,1\} . \\
& L\left(c_{i}\right) \equiv \log \frac{\operatorname{Pr}\left(x_{i}=+1 \mid y_{i}\right)}{\operatorname{Pr}\left(x_{i}=-1 \mid y_{i}\right)}=\log \frac{\operatorname{Pr}\left(c_{i}=0 \mid y_{i}\right)}{\operatorname{Pr}\left(c_{i}=1 \mid y_{i}\right)} \\
& L\left(r_{j i}\right) \equiv \log \frac{r_{j i}(0)}{r_{j i}(1)} \text { and } L\left(q_{i j}\right) \equiv \log \frac{q_{i j}(0)}{q_{i j}(1)}
\end{aligned}
$$




$$
L\left(Q_{i}\right) \equiv \log \frac{Q_{i}(0)}{Q_{i}(1)}
$$

below:

The SPA algorithm involves one initialization step and three iterative steps as shown

Initialization step: Set the initial value of each variable node signal as follows: $L\left(q_{i j}\right) \equiv L\left(c_{i}\right)=2 y_{i} / \sigma^{2}$, where $\sigma^{2}$ is the variance of noise in the AWGN channel.

Iterative steps: The three iterative steps are as follows:

(I) Update check nodes as follows:

$$
L\left(r_{j i}\right)=\left(\prod_{i \in R_{j \backslash i}} \alpha_{i^{\prime} j}\right) \times \phi\left(\sum_{i \in R_{j \backslash i}} \phi\left(\beta_{i^{\prime} j}\right)\right)
$$

Where $\alpha_{i^{\prime} j}=\operatorname{sign}\left(L\left(q_{i j}\right)\right), \beta_{i j}=\left|L\left(q_{i j}\right)\right|$ and $\phi(x)=-\log (\tanh (x / 2))=\log \frac{e^{x}+1}{e^{x}-1}$

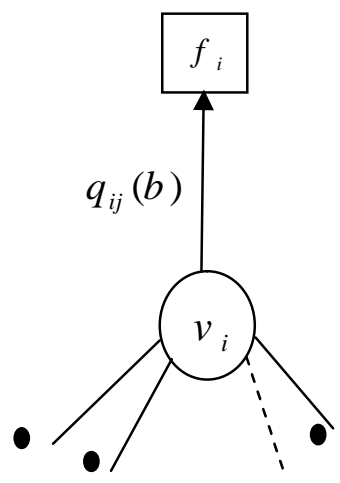

(a)

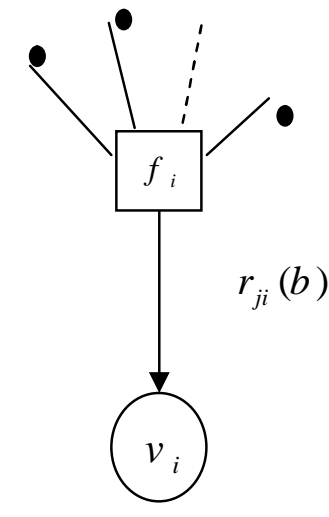

(b)

Fig. 4. (a) Variable-to-check message, (b) Check-to variable message.

(II) Update variable nodes as follows:

$$
L\left(q_{i j}\right)=L\left(c_{i}\right)+\sum_{j \in C_{i j j}} L\left(r_{j^{\prime} i}\right)
$$

(III) Compute estimated variable nodes as follows:

$$
L\left(Q_{i}\right)=L\left(c_{i}\right)+\sum_{j \in C_{i}} L\left(r_{j i}\right)
$$

Based on $L\left(Q_{i}\right)$, the estimated value of the received bit $\left(\hat{c}_{i}\right)$ is given by:

$$
\hat{c_{i}}=\left\{\begin{array}{l}
1 \text { if } L\left(Q_{i}\right) \prec 0 \\
0 \quad \text { else }
\end{array}\right.
$$

During LDPC decoding, the iterative steps I to III are repeated until the following event occurs: $\overline{\hat{c}} \cdot H^{T}=0$ or maximum iterations is reached. This algorithm can be used as hard decision or soft decision.

\subsection{Hamming code}

Hamming codes are the earliest codes capable of actually correcting an error detected at the receiver. However, by definition they are limited so that they can detect and 
correct only a single error. This arises from the fact that minimum hamming distance $=3$, so that if more than one bit is corrupted then the erroneous word is the same as one of the other code words, and the decoding algorithm will assume no error. The Hamming $(7,4)$ code is a famous single error correcting code because it belongs to the class of perfect codes [14]. In the present work, the benefit of using the LDPC as a strong code for coding the ROI and marker while other compressed data are coded with a common Hamming code. This benefit is increasing the image quality and channel noise immunity.

\section{Performance measures}

Image quality metrics are figures of merit used for the evaluation of imaging systems or processes. The image quality metrics can be broadly classified into two categories, subjective and objective which are the performance measures used in evaluating the present work. Subjective image quality is a method of evaluation of images by the viewers and it emphatically examines fidelity and at the same time considers image intelligibility. In objective measures of image quality metrics, some statistical indices are calculated to indicate the reconstructed image quality. The image quality metrics provide some measure of closeness between two digital images by exploiting the differences in the statistical distribution of pixel values. The most commonly used error metrics are Mean Square Error (MSE) and Peak Signal to Noise Ratio (PSNR) [15].

It is useful to know that the human eye does not haveenough sensitivity to detect changes in visual data for PSNRmeasurements above approximately $50 \mathrm{~dB}$, although this mayvary in a minor way for each person. A higher PSNR would normally indicate that the reconstruction is of higher quality(less pixel difference between the images). It is most easily defined via (MSE) which defined as:

$$
\begin{gathered}
\text { MSE }=\frac{1}{m \times n} \sum_{i=0}^{m-1} \sum_{j=0}^{n-1}[I(i, j)-K(i, j)]^{2} \\
\text { PSNR }=10 \times \log _{10}\left(\frac{\text { MAX }^{2}}{\text { MSE }}\right)
\end{gathered}
$$

Where MAX is the maximum possible pixel value of the Image, I or $\mathrm{K}$ is considered as a noisy approximation of the other. $(m \times n)$ is the dimension of the image.

\section{Simulation results}

In this paper subjective and objectiveevaluation of the proposed UEP scheme are carried out and compared with the traditional EEP scheme for the three test images shown in Fig. 5.

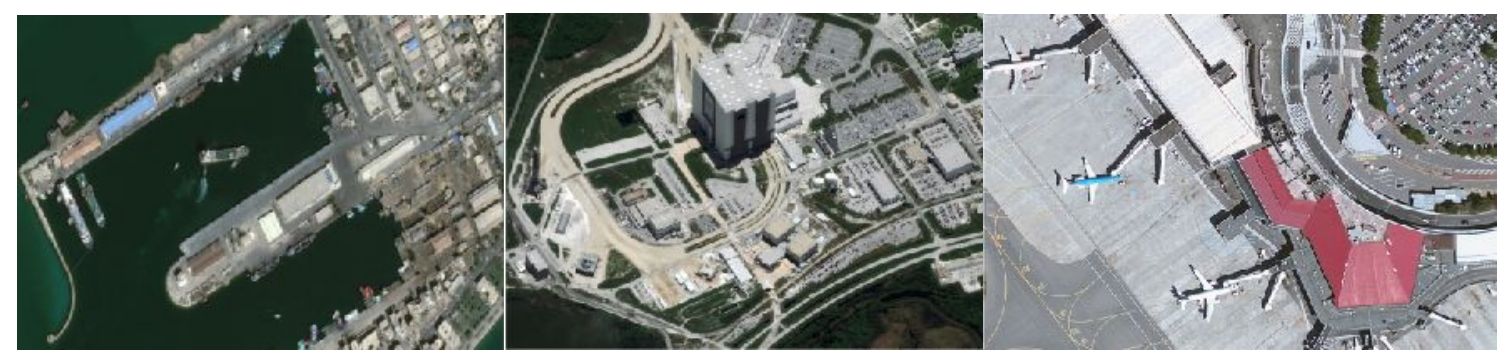

Fig. 5. The three test images used in simulation 
Simulation results were obtained by using Kakadu software V6.4 for compression of JPEG2000 images and assigning the following parameters:

- Three images with size $(\mathrm{m} \times n): 1^{\text {st }}$ image size is $(1024 \times 576), 2^{\text {nd }}$ image size is ( $700 \times 632)$, and $3^{\text {rd }}$ image size is $(997 \times 697)$.

- JPEG2000 image compressed by (5) decomposition levels, (3) layers.

- LDPC code rate $=1 / 2$ for simulation, with number of information bits=32400and block length $=64800$.

- Hamming $(7,4)$ code as simple code.

\subsection{Subjective result}

Subjective results when applying the proposed UEP on the left image of Fig. 6 are shown in Fig. 6. It is clear from this figure that the obtained images by applying the proposed UEP at different SNRs are better than that of the traditional EEP.
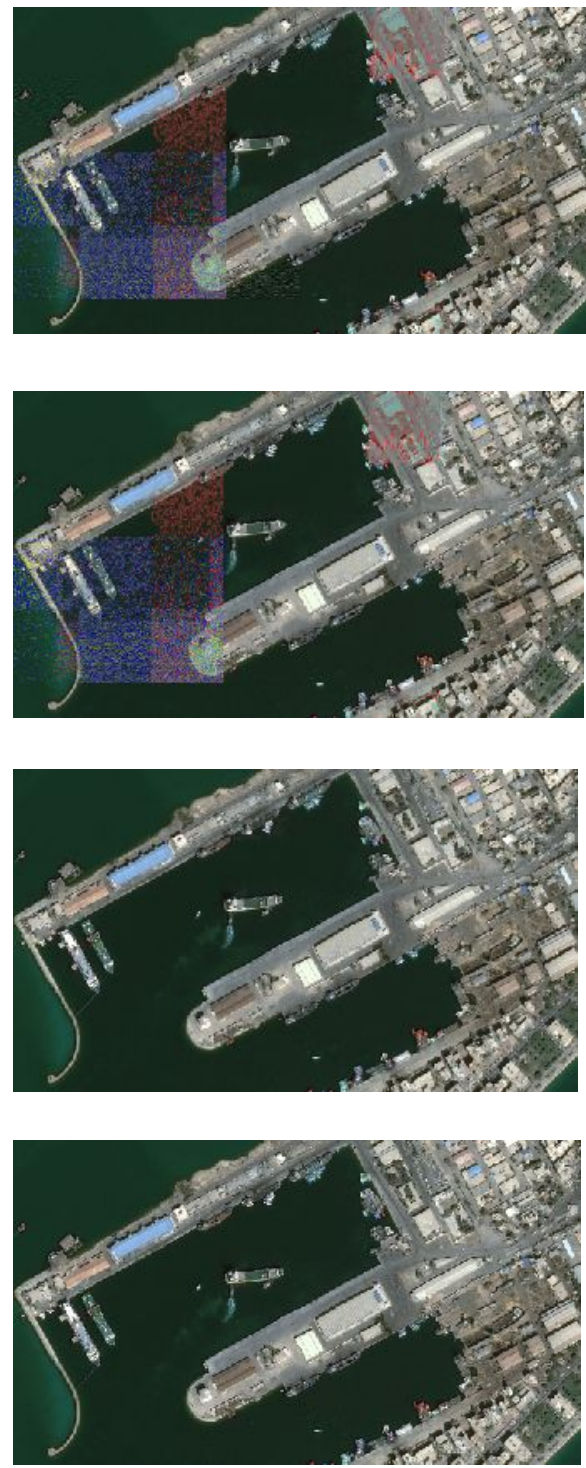

(a)
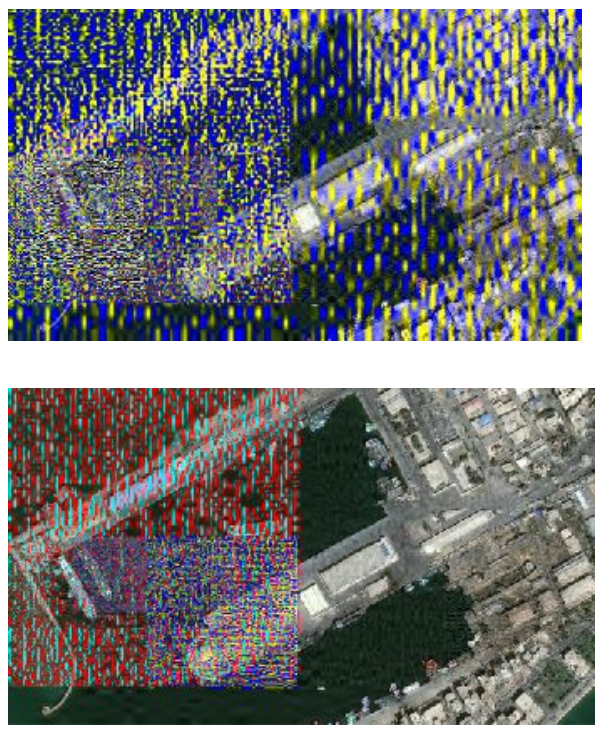

(b)

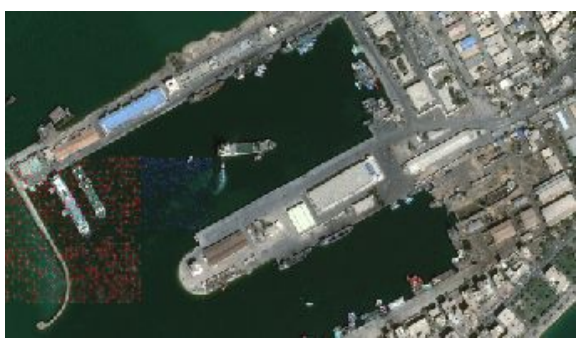

(c)

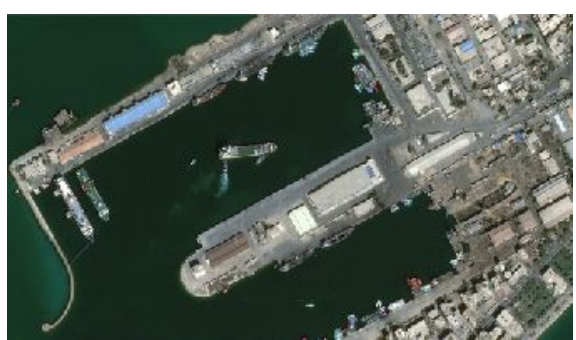

(d) 

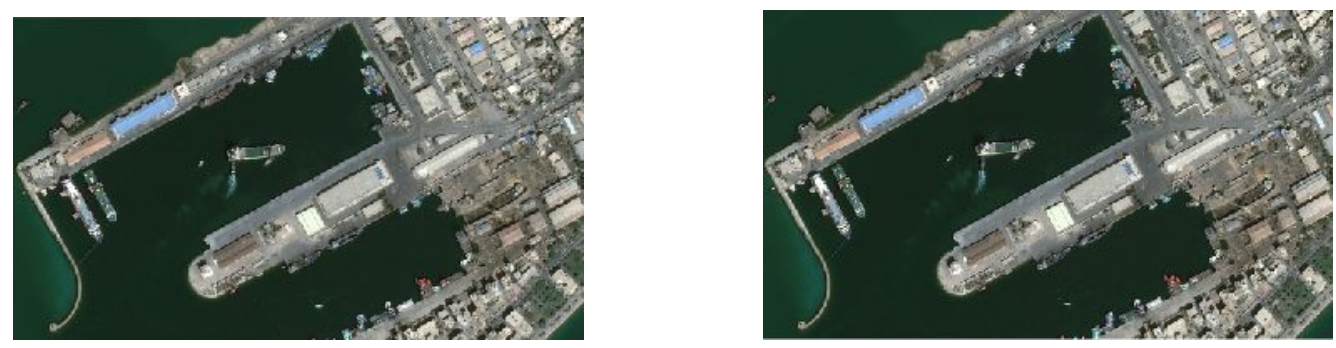

(e)

Fig. 6. Subjective measure results (UEP at left, and EEP at right) at different SNRs; (a) $\mathrm{SNR}=5.5 \mathrm{~dB}$, (b) $\mathrm{SNR}=6 \mathrm{~dB}$, (c) $\mathrm{SNR}=7 \mathrm{~dB}$, (d) $\mathrm{SNR}=8 \mathrm{~dB}$ and (e) $\mathrm{SNR}=8.5 \mathrm{~dB}$

\subsection{Objective result}

The performance ofthe proposed UEP scheme compared to the traditional EEP scheme over the Gaussian channel is shown in Fig. 7.The average value of the resultedPSNR of the three images is plotted against different input SNRs. The figure shows that at approximately 6 $\mathrm{dB}$, the PSNR is above $30 \mathrm{dBwhen}$ using the proposed UEP. So the quality of image is considered to be good while it is below $20 \mathrm{dBfor}$ EEP. PSNR reaches its maximum when using the proposed UEP at $8.5 \mathrm{~dB}$ while it is $9 \mathrm{~dB}$ for EEP.

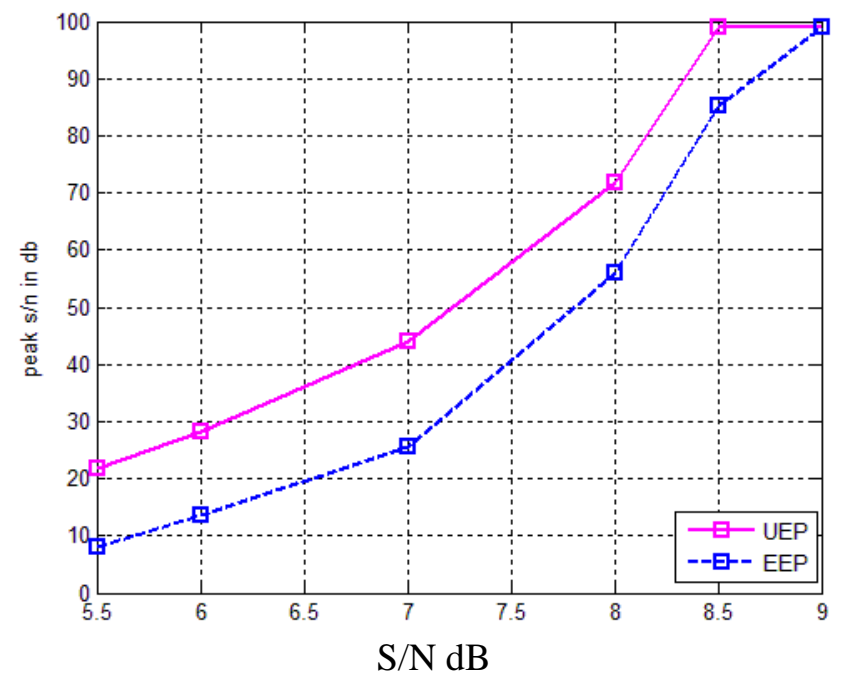

Fig. 7. The average PSNR of the three images when usingEEP and the proposed UEP over the Gaussian channel for different input SNRs.

The Bit Error Rate (BER) is an important factor when analyzing any communication system. In the present work, the BER is calculated for the proposed UEP scheme, the traditional EEP, the LDPC alone, and the uncoded scheme at different $\mathrm{S} / \mathrm{N}$ ratios. These results are shown in Fig. 8. 


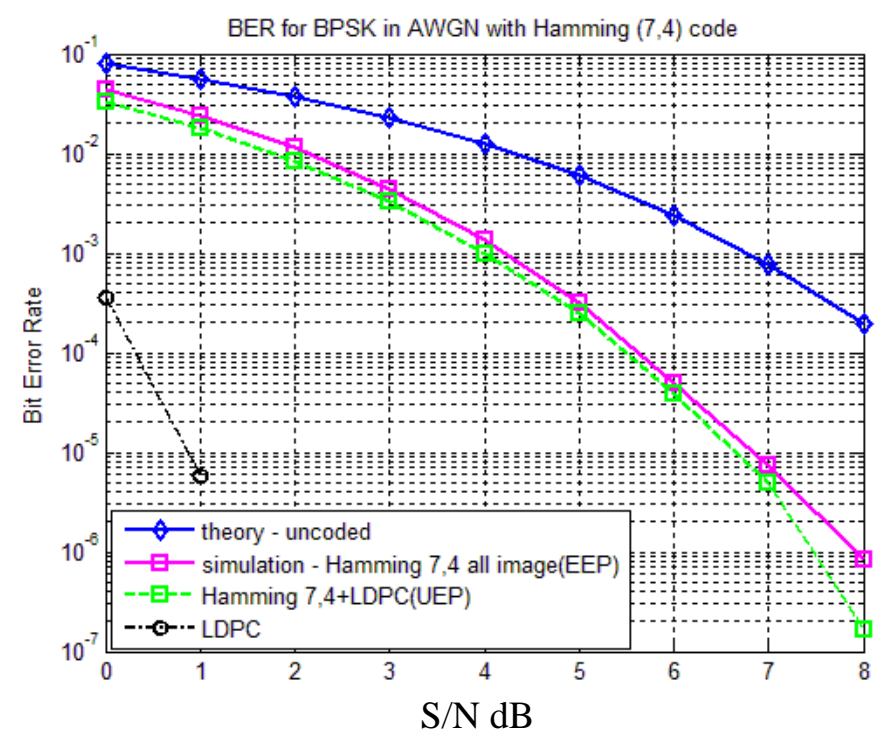

Fig. 8. BER against $\mathrm{S} / \mathrm{N}$ for the proposed UEP, the traditional EEP, LDPC and the theoretical uncoded image.

Fig. 8 shows that the proposed UEP scheme has approximately the same coding gain as the traditional EEP. When calculating the throughput ratio using the proposed UEP, it is found to be 0.552 which is nearly the same as that of the traditional EEP. For the LDPC alone, this ratio will be 0.5 . These results declare that the proposed UEP can give approximately the same coding gain and channel utilization (effective throughput in the channel) as the traditional EEP.

\section{Conclusion}

In the present work an UEP incorporating LDPC for protecting the ROI and headers (markers and marker segments), and Hamming code for protecting compressed data bits was proposed. The superiority of the proposed UEP over traditional EEP was validated objectively and subjectively. The proposed UEP presentedan efficient solution for image transmission over wireless channels, and provided a high quality of service.

\section{References:}

[1]R.Navaneethakrishnan,"Study of Image Compression Techniques", IJSER Volume 3, Issue 7, pp. 1-5,July-2012.

[2] R. Maini and S. Mehra,"A Review on JPEG2000 Image Compression" , International Journal of Computer Applications (0975 - 8887) Volume 11- No.9, December 2010.

[3] A.N. Skodra, C.A. Christopoulos, T. Ebrahimi, «JPEG2000, the upcoming still image compression standard ", Proceeding of the 11th Portuguese Conference on Pattern recognition, Porto, Portugal, May 11th-12th, pp. 359-366, 2000.

[4]P. Schelkens,A. Skodras and T. Ebrahimi"THE JPEG 2000 SUITE",John Wiley \& Sons Inc.,2009.

[5]C. Christopoulos, A. Skodras and T. Ebrahimi, "The JPEG2000 still image coding system: an overview" ,IEEE Trans. on Consumer Electronics, Vol. 46, No. 4, pp. 1103-1127, November 2000. 
[6] K. Kamaras, "JPEG2000 image compression and error resilience for transmission over wireless channels", degree of Master, Naval Postgraduate School, Monterey, March2002.

[7]ISO/IEC JTC1/SC29/WG1/N1646R, "JPEG 2000 Part I final committee draft,version 1.0", March 2000, http://www.jpeg.org/public/fcd15444-1.pdf.

[8]C. Christopoulos, J. Askelöf and M. Larsson, "Efficient methods for encoding regions of interest in the upcoming JPEG2000 still image coding standard", IEEE Signal Processing Letters, Vol. 7, No. 9, pp. 247-249, September 2000.

[9] SandyaRondla, "Unequal Error Protection for Wireless Imaging", Degree of Master, Blekinge Institute of Technology, September 2009.

[10] Y. Qinghua, and C. Miao, "Study of Unequal Error Protection Method Based on JPEG2000 for Surveying \& Mapping ",IEEE Computer Societyin Proc. CSSE (2),pp. 951-954, 2008.

[11] R. Gallager, "Low-density parity-check codes." Ph.D Cambridge, MA, MIT Press, 1963.

[12]William Ryan, "A Low-Density Parity-Check Code Tutorial, Part II - The Iterative Decoder," in ECE dept.: The University of Arizona, April 2002.

[13] C.Nguyen,"Low-Density Parity-Check Codes Construction and Performance Evaluation",degree of Master,The University of New South Wales,Australia,August, 2002.

[14] A. Neuberger, J. Freudenberger and V. Kuhn, "Coding Theory Algorithms, Architectures, and Applications",John Wiley \& Sons Inc., 2007.

[15] BijayShrestha, "JPEG2000: Image Quality Metrics", ASPRS Annual Conference, 2005. 